\title{
Zur Darstellung des Vanadins auf aluminothermischem Wege.
}

\author{
Von
}

R. Voger und G. Tammann.

Die Herren Prandtu und Blexer schlagen zur Reduktion von Vanadinpentoxyd zu Vanadin ein Gemenge von Calcium und Aluminium vor, weil sie mit Aluminium allein, wie auch Gordschmidt und andere kein oxydfreies Vanadin erhalten konnten. Wir haben mit Aluminium allein ein recht reines oxydfreies Vanadin erhalten; allerdings führt die Reduktion von nicht geschmolzenem, trockenen und $\mathrm{NH}_{3}$-freiem Vanadinpentoxyd nicht immer zum Vanadinmetall. Bei Versuchen nach Veröffentlichung unserer Arbeit „Über VanadinEisenlegierungen" erhielten wir aus nicht ermittelten Gründen an Stelle eines guten, metallischen Regulus oxydreiche Schlacken.

Die von Prandte und Bleyer vorgeschlagene Beigabe von Calcium, nach der die Reduktion mit Sicherheit eintreten soll, würde sich gewils empfehlen, wenn das auf diesem Wege gewonnene Vanadin nicbt, wie jene Autoren angeben, nur etwa $94 \%$ Vanadin enthielte. Zur Darstellung von reinem Vanadin bleibt also nur übrig, die Reduktion mit Aluminium allein vorzunehmen, wobei es fürs erste dem Zufall überlassen bleibt, ob man überhaupt ein brauchbares Vanadin oder nur oxydhaltige Schlacken erhält. Das Vanadin, welches von uns aus nicht geschmolzenem Vanadinpentoxyd im mit Magnesia gefütterten Tiegel durch Reduktion mit Aluminium erhalten wurde, enthielt 99.07\% Vanadin. Die Bestimmung des Vanadins wurde nach $H$. Rose ausgeführt. ${ }^{1}$ Aufserdem enthielt das Vanadin noch gegen $1 \%$ Silicium, während Aluminium nicht nachzuweisen war.

Wie man bei der Reduktion von Chromoxyd durch Aluminium nicht selten Fehlversuche zu verzeichnen hat, so kommen solche auch bei der Reduktion von Vanadinpentoxyd vor. Beim Chrom ist diese Schwierigkeit durch Zugabe von $\mathrm{K}_{2} \mathrm{Cr}_{2} \mathrm{O}_{7}$ überwunden worden, beim Vanadin liegen aber Versuche in dieser Richtung nicht vor.

1 Treadwell, Kurzes Lehrbuch der analytischen Chemie II, S. 191.

Göttingen, Institut für physikalische Chemie.

Bei der Redaktion eingegangen am 29. September 1909. 